\title{
Metro Passenger Flow Forecast with a Novel Markov-Grey Model
}

\author{
Yonggang Wang $^{1 *}$, Jingfeng $\mathrm{Ma}^{1}$, Jun Zhang ${ }^{2}$ \\ 1 School of Highway, Chang'an University, P. O. Box 487, Middle Section of Nan'er Huan Rd., Xi'an 710064, Shaanxi, China \\ 2 College of Transportation Engineering, Tongji University, Jiading Campus, 4800 Caoan Rd., Shanghai 201804, China \\ * Corresponding author, e-mail: wangyg@chd.edu.cn
}

Received: 12 June 2018, Accepted: 01 August 2018, Published online: 28 May 2019

\begin{abstract}
Accurate forecasts of passenger flow entering and leaving metro stations is an important work for Metro operation management, such as for the automatic adjustment of train operation diagrams or station passenger crowd regulation planning measures. In this study, Grey theory is introduced to develop a time series GM $(1,1)$ model for total passenger forecasting. Two modification factors determined by two minimum mean square error principles are proposed to decrease the discreteness of input data and thus improve the forecast accuracy. Moreover, the Markov chain approach is further used to optimize the residual error series. Passenger flow data entering and leaving the Xiaozhai station of Xi'an Metro Line 2 from September 1-30, 2015, were utilized to verify the effectiveness of the proposed method; the forecast results show that this novel Markov-Grey model performs well in terms of forecast accuracy with smaller SMSE and MAPE values. To this effect, the proposed method is especially well-suited to smooth passenger flow forecasting compared to other forecast techniques.
\end{abstract}

Keywords

passenger flow forecast, metro station, GM $(1,1)$ model, modification factor, Markov chain

\section{Introduction}

Passenger flow forecasts of mass rail transit (MRT) serve to evaluate and determine the volume of passenger flow at peak hours or within a total day up to a year in the future. The information returned by these forecasts can be utilized for a wide array of applications related to public transit (Lalinská et al., 2017; Zhang et al., 2017). At the planning stage, more importantly, the choice of subway, light rail transit, or streetcar mainly depends on the volume of passenger flow; this affects the preliminary decisions on planning networks as well as the location and design of individual stations and the process of constructing them, including land allocation and supply investment. During the operation period, the passenger's choice of MRT, bus, or other modes of traffic is affected by a variety of uncertain factors including land use type, fees, and convenience of arrival / departure. All of these factors can vary over space and time (Ding et al., 2018; $\mathrm{Ni}$ et al., 2017). In practice, then, it is impossible - or at least very difficult - for forecasters and planners to accurately and comprehensively ascertain the future passenger demands of public transit.
The passenger flow volume of Guangzhou Metro Line 1 was predicted to reach 290,000 in 1998 according to an official assessment, but from its opening day on June 28, 1997, through the end of 1998, the subway carried $175,000 \sim 180,000$ individuals daily - only about $60 \%$ of the expected value. Its total amount of passengers then reached 66,291,300 in 2002, representing annual growth of $0.45 \%$. When the Metro Line 2 opened on June 28, 2003, however, the volume of passenger flow exceeded 1 million, marking an increase of $20 \%$ over the previous year. This figure reached 164 million in 2004. In Xi'an City, the $20.5 \mathrm{~km}$ first section of Metro Line 2 from the North Railway station to the Qujiang International Conference and Exhibition Center was officially opened at midday on September 16, 2011, with services operating at $9.5 \mathrm{~min}$ headways from 6:30 to 21:30; the route's average daily passenger flow volume grew from 151,000 in 2011 to 196,300 before the opening of Metro Line 1 on September 15, 2013. The line now accommodates a passenger volume of approximately 600,000 daily, with a peak of 742,900 passengers on October 1, 2015. Such differences between the 
forecasted and actual values of passenger flow represent serious challenges (e.g., spacious or crowded platforms and carriages) to the operating management, thus necessitating more reliable and more accurate approaches to passenger flow forecasting (Li, X. et al., 2018; Zhang et al., 2017).

Previous researchers have established several approaches to passenger flow forecasting, but from a more general perspective (Zhao et al., 2011). For example, data sequences are generally used and mathematical modeling is generally the focus in developing these techniques (Leng et al., 2013; Li, L. et al., 2018), but they are lacking in accuracy overall. Grey system theory is a multidisciplinary theory that was firstly proposed in early 1980s by Deng to describe systems such as passenger flow (Deng, 1982); the theory has indeed proven superior to conventional statistical models in dealing with systems that have both partially known and partially unknown parameters (Liu et al., 2011). Over recent decades, Grey system theory has garnered research attention across a variety of fields and has been successfully applied to systems such as economy, finance, transportation, mechanics, geology, military, and medical care (Carmona Benítez et al., 2013; Yin et al., 2017; Zhou and $\mathrm{He}, 2013)$. The GM $(1,1)$ model is the main forecasting model applicable to Grey theory, and has achieved satisfactory results in field use though its forecasting precision and applicability merit further improvement.

Two of the deficiencies in the methods described above constituted the primary motivation of this paper. First, explored an approach to improving the accuracy of the general GM $(1,1)$ model in forecasting the passenger flow of mass rail transit; and second, we attempted to combine the Markov process with the proposed Grey model to enhance forecasting efficiency. The remainder of this paper is structured as follows: Section 2 describes our process in designing the proposed Markov-Grey method, and Section 3 presents its application in forecasting the passenger flow of Xiaozhai station of Xi'an Metro Line 2. Concluding remarks are provided in Section 4.

\section{Methodology}

\subsection{Grey forecast model}

Let an original non-negative time series with $n$ records be $x^{(0)}=\left[x^{(0)}(1), x^{(0)}(2), \ldots, x^{(0)}(n)\right]$, then the monotonically increasing sequence $x^{(1)}$ can be cumulatively generated as $\quad x^{(1)}=\left[x^{(1)}(1), x^{(1)}(2), \ldots, x^{(1)}(n)\right]$, where $\quad x^{(1)}(1)=x^{(0)}(1) \quad$ and $\quad x^{(1)}(k)=\sum x^{(0)}(i)$, $i=1,2, \ldots, k(k=2, \ldots, n)$. The raw data $x^{(0)}$ are then ranked as $\sigma=[\sigma(2), \sigma(3), \ldots, \sigma(n)]$, where $\sigma(k)=x^{(0)}(k-1) / x^{(0)}(k), k=2,3, \ldots, n$, and satisfy the Grey model if the sequence falls within the range 0.1345-7.389 (Deng, 1982).

According to Grey theory, the original Grey model can be expressed as $x^{(0)}=-a z^{(1)}(k)+b, k=2,3, \ldots, n$, where $z^{(1)}(k)=\left[x^{(1)}(k)+x^{(1)}(k-1)\right] / 2$. This differential equation is:

$\frac{d x^{(1)}(k)}{d t}+a x^{(1)}(k)=b$

with initial condition $x^{(1)}(1)=x^{(0)}(1)$; such replacement is defined as "whiteness processing" (Deng, 1982). Optimal coefficients $a$ and $b$ can both be estimated by the minimum least square method as $[a, b]^{\mathrm{T}}=\left(B^{\mathrm{T}} B\right)^{-1} B^{\mathrm{T}} Y$, where $B$ is the accumulated matrix and $Y$ is the constant term vector (Deng, 1982), and then substituted into the differential equation to obtain the approximate relation for $k=2,3, \ldots, n$ :

$\hat{x}^{(1)}(k)=\left[x^{(0)}(1)-\frac{b}{a}\right] e^{-a(k-1)}+\frac{b}{a}$

where $\hat{x}^{(1)}(1)=x^{(0)}(1)$, for $k=2,3, \ldots, n$. The forecast value of the series can be restored via the following inverse accumulated generating operation:

$\hat{x}^{(0)}(k)=\left(1-e^{a}\right)\left[x^{(0)}(1)-\frac{b}{a}\right] e^{-a(k-1)}$.

Precision checking was conducted to determine the residual errors $e(k)$ between actual $x^{(0)}(k)$ and predicted $\hat{x}^{(0)}(k)$, respectively, as $\left[x^{(0)}(k)-\hat{x}^{(0)}(k)\right] \times 100 \% / x^{(0)}(k)$; the precision index was calculated to be $1-e(k)$. We utilized the Standard Mean Square Error (SMSE) and the Mean Absolute Percentage Error (MAPE) to verify the performance of residual error $e(k)$ as given by Eqs. (4), (5). Here, the acceptable MAPE was set to be 0.2 for daily passenger flow forecasting and an average precision of 1-MAPE exceeding $80 \%$ was considered to indicate excellent model forecast efficiency.

$$
\begin{aligned}
& \text { SMSE }=\sqrt{\frac{1}{n-1} \sum_{k=2}^{n}\left[x^{(0)}(k)-\hat{x}^{(0)}(k)\right]^{2}} \\
& \text { MAPE }=\frac{1}{n-1} \sum_{k=2}^{n}\left|\frac{x^{(0)}(k)-\hat{x}^{(0)}(k)}{x^{(0)}(k)}\right| \times 100 \% .
\end{aligned}
$$

\subsection{Precision modification}

According to Grey theory, forecast accuracy is affected significantly by two parameters: $a$ and $b$. Their solution depends on the background value. In establishing the $\mathrm{GM}(1,1)$ model, $z^{(1)}(k) \cdot z^{(1)}(k)=\alpha x^{(1)}(k)+(1-\alpha) x^{(1)}(k-1)$ was 
considered as the smoothing value, where $\alpha$ is the weighting factor, and $\alpha=0.5$ is chosen as a special case but may not be the optimal result (Hsu and Chen, 2003). When the original data sequence $x^{(0)}(k)$ changes smoothly, the proposed GM $(1,1)$ model yields more accurate forecast results (Hui et al., 2013). Therefore, the forecast data $\hat{x}^{(0)}(k)$ requires further modification. Suppose the approximate relation of $\hat{x}^{(0)}(k)$ is modified as follows:

$$
\tilde{x}^{(1)}(k)=\left[x^{(0)}(1)+\delta-\frac{b}{a}\right] e^{-a(k-1)}+\frac{b}{a}
$$

and thus reaches $\tilde{x}^{(0)}(k)=\hat{x}^{(0)}(k)+\delta\left(1-e^{a}\right) e^{-a(k-1)}$, $k=2,3, \ldots, n$, where $\delta$ is the modification factor. Two criteria are proposed here to determine the value of $\delta$.

CRITERIA 1: Minimum mean square error forecast of cumulative data series

$$
\min _{\delta_{1}}\left[\tilde{x}^{(1)}(k)-x^{(1)}(k)\right]^{2} \text {. }
$$

Since $\tilde{x}^{(1)}(k)=\left[x^{(1)}(1)+\delta_{1}-\frac{b}{a}\right] e^{-a(k-1)}+\frac{b}{a}$, then

$\varphi_{1}=\sum_{k=2}^{n}\left\{\left[x^{(k)}(1)+\delta_{1}-\frac{b}{a}\right] e^{-a(k-1)}+\frac{b}{a}-x^{(1)}(k)\right\}^{2}$.

If $\frac{\partial \varphi_{1}}{\partial \delta_{1}}=0$, then

$\left[x^{(0)}(1)-\frac{b}{a}\right] \sum_{k-2}^{n} e^{-2 a(k-1)}+\delta_{1} \sum_{k=2}^{n} e^{-2 a(k-1)}-\sum_{k=2}^{n} x^{(1)}(k) e^{-a(k-1)}$

$=0$.

It is clear that $\sum_{k=2}^{n} \mathrm{e}^{-2 a(k-1)}=\frac{1-\mathrm{e}^{-2 n a}}{1-\mathrm{e}^{-2 a}}$, hence, by Eq. (9):

$\delta_{1}=\frac{\sum_{k=2}^{n} x^{(1)}(k) e^{-a(k-1)}}{\frac{1-e^{-2 n a}}{1-e^{-2 a}}}-\frac{b}{a} \cdot \frac{\frac{1-e^{-n a}}{1-e^{-a}}}{\frac{1-e^{-2 n a}}{1-e^{-2 a}}}\left[x^{(0)}(1)-\frac{b}{a}\right]$.

CRITERIA 2: Minimum mean square error forecast of original data series

$\min _{\delta_{2}}\left[\tilde{x}^{(0)}(k)-x^{(0)}(k)\right]^{2}$.

Inserting $\tilde{x}^{(0)}(k)=\left(1-e^{a}\right)\left[x^{(0)}(1)+\delta_{2}-\frac{b}{a}\right] e^{-a(k-1)}$ into Eq. (11) yields:

$\varphi_{2}=\sum_{k=2}^{n}\left\{\left(1-e^{a}\right)\left[x^{(0)}(1)+\delta_{2}-\frac{b}{a}\right] e^{-a(k-1)}-x^{(0)}(k)\right\}^{2}$.
Let $\frac{\partial \varphi_{2}}{\partial \delta_{2}}=0$, then

$\left(1-e^{a}\right) \cdot\left[x^{(0)}(1)-\frac{b}{a}\right] \sum_{k=2}^{n} e^{-2 a(k-1)}+\delta_{2}\left(1-e^{a}\right) \sum_{k=2}^{n} e^{-2 a(k-1)}$

$-\sum_{k=2}^{n} x^{(0)}(k) e^{-a(k-1)}=0$.

Because $\sum_{k=2}^{n} e^{-2 a(k-1)}=\frac{1-e^{-2 n a}}{1-e^{-2 a}}, \delta_{2}$ can be rewritten as:

$\delta_{2}=\frac{\sum_{k=2}^{n} x^{(0)}(k) e^{-a(k-1)}}{\left(1-e^{a}\right) \frac{1-e^{-2 n a}}{1-e^{-2 a}}}-\left[x^{(0)}(1)-\frac{b}{a}\right]$.

Using $\delta_{1}$ and $\delta_{2}$, therefore, we obtained two improved GM $(1,1)$ models by plus $\delta_{i}\left(1-e^{a}\right) e^{-a(k-1)}(k=2,3, \ldots, n)$, respectively. The optimal model (with relatively fewer errors) was then used for the remainder of the forecasting process.

\subsection{Markov process}

To reduce forecast errors, Markov approach is integrated into the model described above to process the forecast error series $\{e(k), k=2,3, \ldots, n\}$. Let $e(k)$ be divided into $q$ states $\left(R_{1}, R_{2}, \ldots, R_{q}\right)$ by appealing to the equiprobability principle, and $E_{l i}$ and $E_{u i}$ represent the lower and upper bounds of each state, respectively. Let current state be $R_{i}$ and another state be $R_{j}$, then the transition probability $P_{i j}$ from state $R_{i}$ to $R_{j}$ after $w$ steps is written as $P_{i j}^{(w)}=m_{i j}^{(w)} / M_{i}, i, j=1,2, \ldots, q$, where $m_{i j}^{(w)}$ is the frequency of transition from state $R_{i}$ to $R_{j}$ in $w$ steps and $M_{i}$ is the value of state $R_{i}$ (Samet and Mojallal, 2014). Thus, the transition probability matrix can be expressed as:

$P^{(w)}=\left[\begin{array}{cccc}p_{11}^{(w)} & p_{12}^{(w)} & \cdots & p_{1 q}^{(w)} \\ p_{21}^{(w)} & p_{22}^{(w)} & \cdots & p_{2 q}^{(w)} \\ \vdots & \vdots & \vdots & \vdots \\ p_{q 1}^{(w)} & p_{q 2}^{(w)} & \cdots & p_{q q}^{(w)}\end{array}\right]$.

Considering $w=1$, if the residual errors $e(k)$ falls in the $i^{\text {th }}$ state, then check the $i^{\text {th }}$ row of transition probability matrix $P^{(1)}$. If $\operatorname{Max} P_{i j}^{(1)}=P_{i L}$, then state $L$ is likely to be the next transition of forecast state (Samet and Mojallal, 2014). The forecast residual error value $e(k)$ can be modified into $e(k)-\left(A_{k}+C_{k}\right) / 2$ by the lower and upper bounds of $A_{L}$ and $C_{L}$. Subsequently, the forecast value can be appropriately modified. 


\section{Illustrative Example and Analysis}

The first and second phases of Xi'an Metro Line 2 were officially opened on September 16, 2011, and June 16, 2014, respectively. The line runs between the new North Railway station and Weiqunan along a straight northsouth corridor passing directly through the city centre, covering the Administrative Centre, Bell Tower commercial area, Xiaozhai, Qujiang International Conference and Exhibition Center, and other important passenger distribution centres. It is currently $26.8 \mathrm{~km}$ long with 21 stations and is known to provide a safe, comfortable, convenient, and swift transportation service for about 600,000 passengers from 6:00 a.m. to 11:10 p.m. daily.

As discussed in the Introduction, passenger flow volume forecasting has long been regarded as a critical requirement for mass rail transit scheduling and operations management (Leng et al., 2013; Li, L. et al., 2018). We selected Xiaozhai station, a major transfer station of Metro Line 2, as a case study and gathered its daily passenger flow data for
September, 2015. Each record in the data set provided here refers to the number of passengers entering and leaving this station. The sample data 20150901-20150922 were used for model fitting, as shown in Table 1, and 20150923-20150930 were reserved for forecasting and accuracy testing.

The primary data sequence $x^{(0)}$ is given as [38745, 43633, ... 42124], and the one-order cumulative sequence is generated as $x^{(1)}=[38745,82378, \ldots, 1069172]$. The $\sigma$ series is then $[0.8880,0.9911, \ldots, 1.4551]$, with values ranging between 0.1345 and 7.389 ; this indicates that the selected sample data are suitable for constructing the Grey forecast model for passenger flow in Xiaozhai metro station.

Two parameters, $a$ and $b$, were estimated by minimum least square method as -0.0057 and 45957.7945 , respectively, yielding an original GM $(1,1)$ model expressed as $\hat{x}^{(0)}(k)=46047.2816 e^{0.0057(k-1)}, k=2,3, \ldots, n$ with the initial condition $\hat{x}^{(0)}(1)=x^{(0)}(1)=38745$. After substituting $k=2,3, \ldots, n$ into Eq. (1), the forecast data of passenger flows from 20150902 to 20150922 were obtained as well

Table 1 The observed and forecasted passenger flow using three GM(1,1) models

\begin{tabular}{|c|c|c|c|c|c|c|c|c|}
\hline \multirow{2}{*}{ Data } & \multirow{2}{*}{$\begin{array}{c}\text { Passengers } \\
x^{(0)}\end{array}$} & \multicolumn{2}{|c|}{$\mathrm{GM}(1,1)$} & \multicolumn{3}{|c|}{$\operatorname{GM}(1,1)$ with $\delta_{1}$} & \multicolumn{2}{|c|}{$\operatorname{GM}(1,1)$ with $\delta_{2}$} \\
\hline & & $\hat{x}^{(0)}$ & $e / \%{ }^{1}$ & $\tilde{x}^{(0)}$ & $e / \%{ }^{2}$ & State & $\tilde{x}^{(0)}$ & $e / \%{ }^{3}$ \\
\hline $20150901^{4}$ & 38745 & 38745 & - & 38745 & - & - & 38745 & - \\
\hline 20150902 & 43633 & 46311 & -6.14 & 44104 & -1.08 & 3 & 44462 & -1.90 \\
\hline 20150903 & 44026 & 46575 & -5.79 & 45004 & -2.22 & 3 & 44716 & -1.57 \\
\hline 20150904 & 43497 & 46841 & -7.69 & 44978 & -3.40 & 3 & 44972 & -3.39 \\
\hline 20150905 & 54755 & 47109 & 13.96 & 57006 & -4.11 & 5 & 45229 & 17.40 \\
\hline 20150906 & 63344 & 47379 & 25.20 & 66489 & -4.97 & 6 & 45488 & 28.19 \\
\hline 20150907 & 49411 & 47649 & 3.57 & 52824 & -6.91 & 5 & 45748 & 7.41 \\
\hline 20150908 & 54040 & 47922 & 11.32 & 58236 & -7.76 & 5 & 46009 & 14.86 \\
\hline 20150909 & 37999 & 48196 & -26.83 & 42101 & -10.79 & 1 & 46272 & -21.77 \\
\hline 20150910 & 39227 & 48471 & -23.57 & 43861 & -11.81 & 2 & 46537 & -18.63 \\
\hline 20150911 & 41868 & 48748 & -16.43 & 47144 & -12.60 & 2 & 46803 & -11.79 \\
\hline 20150912 & 49486 & 49027 & 0.93 & 55778 & -12.71 & 4 & 47070 & 4.88 \\
\hline 20150913 & 60949 & 49307 & 19.10 & 68672 & -12.67 & 6 & 47339 & 22.33 \\
\hline 20150914 & 51189 & 49589 & 3.13 & 58927 & -15.12 & 4 & 47610 & 6.99 \\
\hline 20150915 & 39448 & 49872 & -26.42 & 46863 & -18.80 & 1 & 47882 & -21.38 \\
\hline 20150916 & 40840 & 50158 & -22.82 & 48891 & -19.71 & 2 & 48156 & -17.91 \\
\hline 20150917 & 43009 & 50444 & -17.29 & 51801 & -20.44 & 2 & 48431 & -12.61 \\
\hline 20150918 & 44328 & 50733 & -14.45 & 53820 & -21.41 & 3 & 48708 & -9.88 \\
\hline 20150919 & 54049 & 51023 & 5.60 & 65178 & -20.59 & 4 & 48986 & 9.37 \\
\hline 20150920 & 71911 & 51314 & 28.64 & 85798 & -19.31 & 6 & 49266 & 31.49 \\
\hline 20150921 & 61294 & 51608 & 15.80 & 74847 & -22.11 & 5 & 49548 & 19.16 \\
\hline 20150922 & 42124 & 51903 & -23.21 & 54060 & -28.34 & 2 & 49831 & -18.30 \\
\hline
\end{tabular}


as the residual errors $e(k)$ between $x^{(0)}(k)$ and $\hat{x}^{(0)}(k)$ (see Table 1 ). The values of $e(k)$ vary between $-26.83 \%$ and $28.64 \%$, and the corresponding SMSE and MAPE are 8913.42 and $15.14 \%$, respectively.

Modification factor $\delta$ was introduced to improve the forecasting performance of the original GM $(1,1)$ model. Criteria 1 and 2 yield $\delta_{1}=520646.1540$ and $\delta_{2}=-323352.0908$, respectively, thus the proposed GM $(1,1)$ model is improved into $\tilde{x}^{(0)}(k)=49006.5228 e^{0.0057(k-1)}$ and $\tilde{x}^{(0)}(k)=44209.4176 e^{0.0057(k-1)}$ for $k=2,3, \ldots, n$. We obtained the data sequence of $\tilde{x}^{(0)}(k)$ by substituting $k=2,3, \ldots, n$ into the two improved forecasting models. These results as well as the corresponding SMSE and $M A P E$ values are presented in Table 1 . We conclude, accordingly, that $\tilde{x}^{(0)}(k)=49006.5228 e^{0.0057(k-1)}$ is a well-fitted forecast model.

These error series were further divided into six states: State 1 for $-\infty--20 \%$, state 2 for $-20 \%--10 \%$, state 3 for state 2 for $-10 \%-0$, state 4 for $0 \%-10 \%$, state 5 for $10 \%-20 \%$, and state 6 for $20 \%-+\infty$, according to the precision requirements for forecasting. The state divisions of error series $\{-1.08,-2.22, \ldots,-28.34\}$ are given in Table 1. The state matrix $S$ and one-step transition probability matrix $P^{(1)}$ are:

$S=\left[\begin{array}{llllll}0 & 2 & 0 & 0 & 0 & 0 \\ 0 & 2 & 1 & 1 & 0 & 0 \\ 0 & 0 & 2 & 1 & 1 & 0 \\ 1 & 0 & 0 & 0 & 0 & 2 \\ 1 & 1 & 0 & 0 & 1 & 1 \\ 0 & 0 & 0 & 1 & 2 & 0\end{array}\right]$ and

$$
P^{(1)}=\left[\begin{array}{llllll}
0 & 1 & 0 & 0 & 0 & 0 \\
0 & 0.5 & 0.25 & 0.25 & 0 & 0 \\
0 & 0 & 0.5 & 0.25 & 0.25 & 0 \\
0.33 & 0 & 0 & 0 & 0 & 0.67 \\
0.25 & 0.25 & 0 & 0 & 0.25 & 0.25 \\
0 & 0 & 0 & 0.33 & 0.67 & 0
\end{array}\right] .
$$

The new time series of $k=23,24, \ldots, 30$ were then substituted into the general $\operatorname{GM}(1,1)$ model and novel GM $(1,1)$ model improved by modification factor $\delta_{1}$ to obtain the forecast values and performance indicators, which were then compared against the statistical data \{59602, 53752, 60297, 59195, 58567, 50608, 56664, $60760\}$ as shown in Table 2. The error series from the novel Grey forecast model were further processed via Markov approach. For example, the error 6.79 of 201509230 data remains at state 4 and 0.67 is the maximum element in row 4 of $P^{(1)}$. So, this error is most likely to turn to state 6 associated with residual error of $-5.10 \%$. Clearly, the MarkovGrey model exhibits remarkable forecasting accuracy with relatively small SMSE and MAPE values.

\section{Conclusion}

Metro passenger flow forecasting is an estimation of the future traffic demand for a certain station, metro line, or overall metro network. Quick, comprehensible forecasts are required to provide sufficient travel information for passengers, and to allow relevant personnel to adjust train operation diagrams dynamically; accurate forecast information also allows personnel to make effective emergency management decisions for stations encountering

Table 2 Performance comparison of passenger flow forecast

\begin{tabular}{|c|c|c|c|c|c|c|c|}
\hline \multirow{2}{*}{ Data } & \multirow{2}{*}{$\begin{array}{c}\text { Passengers } \\
x^{(0)}\end{array}$} & \multicolumn{2}{|c|}{$\mathrm{GM}(1,1)$} & \multicolumn{2}{|c|}{$\operatorname{GM}(1,1)$ with $\delta_{1}$} & \multicolumn{2}{|c|}{ Markov-GM(1,1) with $\delta_{1}$} \\
\hline & & $\hat{x}^{(0)}$ & $e / \%{ }^{1}$ & $\tilde{x}^{(0)}$ & $e / \%{ }^{2}$ & $\tilde{x}^{(0)}$ & $e / \%{ }^{3}$ \\
\hline 20150923 & 58602 & 52199 & 10.93 & 55554 & 5.20 & -4 & - \\
\hline 20150924 & 59752 & 52498 & 12.14 & 55871 & 6.49 & 61849 & -3.51 \\
\hline 20150925 & 60297 & 52798 & 12.44 & 56191 & 6.81 & 62220 & -3.19 \\
\hline 20150926 & 62195 & 53100 & 14.62 & 56512 & 9.14 & 62730 & -0.86 \\
\hline 20150927 & 58067 & 53403 & 8.03 & 56835 & 2.12 & 61714 & -6.28 \\
\hline 20150928 & 61608 & 53708 & 12.82 & 57160 & 7.22 & 63321 & -2.78 \\
\hline 20150929 & 63164 & 54015 & 14.48 & 57487 & 8.99 & 63802 & -1.01 \\
\hline 20150930 & 60986 & 54324 & 10.92 & 57815 & 5.20 & 63913 & -4.80 \\
\hline
\end{tabular}


an outburst of passengers. Passenger flow forecasting is often largely inaccurate due to various uncertainties (Li, L. et al., 2018; Zhao et al., 2011) that merit special consideration in ATC application.

In this paper, we simultaneously propose a passenger flow forecast method comprised of a novel Markov-Grey model which includes modification factors $\delta_{1}$ and $\delta_{2}$, as determined by two minimum mean square error principles, to improve the forecast precision; the Markov approach is utilized to further process the residual error series. Flow data for passengers entering and leaving the Xiaozhai station of Xi'an Metro Line 2 from September 1-30, 2015, were used to construct the Grey forecast model. Our analysis results revealed that the proposed method has better forecasting performance, with smaller SMSE and MAPE values than the original method; Markov processing further decreases the residual errors.

\section{References}

Carmona Benítez, R. B., Carmona Paredes, R. B., Lodewijks, G., Nabais, J. L. (2013) "Damp trend Grey Model forecasting method for airline industry", Expert Systems with Applications, 40(12), pp. 4915-4921. https://doi.org/10.1016/j.eswa.2013.02.014

Deng, J.-L. (1982) "Control problems of grey system", Systems \& Control Letters, 1(5), pp. 288-294. https://doi.org/10.1016/S0167-6911(82)80025-X

Ding, X., Guan, S., Sun, D. J., Jia, L. (2018) "Short turning pattern for relieving metro congestion during peak hours: the substance coherence of Shanghai, China", European Transport Research Review, 10(2), article number: 28. https://doi.org/10.1186/s12544-018-0293-9

Hsu, C.-C., Chen, C.-Y. (2003) "Applications of improved grey prediction model for power demand forecasting", Energy Conversion and Management, 44(14), pp. 2241-2249.

https://doi.org/10.1016/S0196-8904(02)00248-0

Hui, H., Li, F., Shi, Y. (2013) "An Optimal Multi-variable Grey Model for Logistics Demand Forecast", International Journal of Innovative Computing, Information and Control, 9(7), pp. 2907-2918.

Lalinská, J., Gašparík, J., Šipuš, D. (2017) "Factors Affecting the Delay of Passenger Trains", LOGI-Scientific Journal on Transport and Logistics, 8(1), pp. 74-81. https://doi.org/10.1515/logi-2017-0009

Leng, B., Zeng, J., Xiong, Z., Lv, W., Wan, Y. (2013) "Probability tree based passenger flow prediction and its application to the Beijing subway system", Frontiers of Computer Science, 7(2), pp. 195-203. https://doi.org/10.1007/s11704-013-2057-y

Li, L., Wang, Y., Zhong, G., Zhang, J., Ran, B. (2018) "Short-to-medium Term Passenger Flow Forecasting for Metro Stations using a Hybrid Model", KSCE Journal of Civil Engineering, 22(5), pp. 1937-1945. https://doi.org/10.1007/s12205-017-1016-9

Liu, S., Forrest, J., Yang, Y. (2011) "A brief introduction to grey system theory", Grey Systems: Theory and Application, 2(2), pp. 89-104. https://doi.org/10.1108/20439371211260081
This study arguably has some methodological limitations. For one, because the Grey forecast model is the form of exponential function, better forecasting performance would be possible if the original input $\left\{x^{(0)}\right\}$ strictly increases (or decreases) the time series data; but the data series would yield large residual errors if $\left\{x^{(0)}\right\}$ is harsh. The model proposed here also does not include the stochastic characteristics of metro passenger flow series. In future work, we plan to extend this methodology to incorporate the uncertainty of metro passenger flow forecasting using a fuzzy rule-based approach.

\section{Acknowledgement}

This research is financially supported by the National Natural Science Foundation of China (No. 71871027). The authors also would like to thank the Xi'an Metro Co., Ltd. for help with data collection.

Li, X., Chen, P., Chen, F., Wang, Z. (2018) "Passenger flow analysis of Beijing urban rail transit network using fractal approach", Modern Physics Letters B, 32(10), article number: 1850001. https://doi.org/10.1142/S021798491850001X

Ni, M., He, Q., Gao, J. (2017) "Forecasting the Subway Passenger Flow Under Event Occurrences With Social Media", IEEE Transactions on Intelligent Transportation Systems, 18(6), pp. 1623-1632. http://doi.org/10.1109/TITS.2016.2611644

Samet, H., Mojallal, A. (2014) "Enhancement of electric arc furnace reactive power compensation using Grey-Markov prediction method", IET Generation, Transmission \& Distribution, 8(9), pp. 1626-1636. https://doi.org/10.1049/iet-gtd.2013.0698

Yin, K., Lu, D., Li, X. (2017) "A Novel Grey Wave Method for Predicting Total Chinese Trade Volume", Sustainability, 9(12), article number: 2367.

https://doi.org/10.3390/su9122367

Zhang, J., Shen, D., Tu, L., Zhang, F., Xu, C., Wang, Y., Tian, C., Li, X., Huang, B., Li, Z. (2017) "A Real-Time Passenger Flow Estimation and Prediction Method for Urban Bus Transit Systems", IEEE Transactions on Intelligent Transportation Systems, 18(11), pp. $3168-3178$.

http://doi.org/10.1109/TITS.2017.2686877

Zhao, S.-Z., Ni, T.-H., Wang, Y., Gao, X.-T. (2011) "A new approach to the prediction of passenger flow in a transit system", Computers and Mathematics with Applications, 61(8), pp. 1968-1974. https://doi.org/10.1016/j.camwa.2010.08.023

Zhou, W., He, J.-M. (2013) "Generalized GM $(1,1)$ model and its application in forecasting of fuel production", Applied Mathematical Modelling, 37(9), pp. 6234-6243. https://doi.org/10.1016/j.apm.2013.01.002 